\title{
EFEITO DA ADIÇÃO DE LODO DE CURTUME NA FERTILIDADE DO SOLO, NODULAÇÃO E RENDIMENTO DE MATÉRIA SECA DO CAUPI
}

\author{
Effect of tannery sludge addition on soil fertility, nodulation and dry matter yield of Cowpea
}

\author{
Keyla Rafaelly Gramosa Teixeira ${ }^{1}$, Luiz Alberto Ribeiro Gonçalves Filho', \\ Eulália Maria Sousa Carvalho², Ademir Sérgio Ferreira de Araújo ${ }^{3}$, Valdinar Bezerra dos Santos ${ }^{4}$
}

\begin{abstract}
RESUMO
O objetivo deste trabalho foi avaliar o efeito do lodo de curtume sobre a fertilidade do solo, a nodulação e rendimento de matéria seca do caupi. O estudo foi conduzido em casa-de-vegetação, utilizando-se lodo de curtume adicionado ao solo, sendo em seguida plantadas sementes de caupi inoculadas com Bradyrhizobium sp. Os dados foram coletados aos 35 e 49 dias após a emergência das plantas. $\mathrm{O}$ lodo de curtume elevou o $\mathrm{pH}$ e os teores de matéria orgânica, cálcio e sódio do solo. Além disso, houve aumento na salinidade do solo com a aplicação do resíduo. Por outro lado, houve um incremento no rendimento de matéria seca do caupi com a adição do lodo de curtume, embora, em altas doses, o resíduo tenha diminuído a nodulação do caupi pela estirpe de Bradyrhizobium $s p$ inoculada.
\end{abstract}

Termos para indexação: Bradyrhizobium, indicador biológico, Vigna unguiculata.

\begin{abstract}
The aim of this paper was to evaluate the effect of tannery sludge addition on soil fertility, nodulation and dry matter yield by cowpea. The study was conducted in greenhouse using tannery sludge applied to soil, and cowpea seeds inoculated with Bradyrhizobium $s p$. Data were collected at 35 and 49 days after plant emergence. Tannery sludge increased soil $\mathrm{pH}$ and organic matter, calcium and sodium content. There was an increase in soil salinity with application of the residue. There was also an increase in the dry matter yield of cowpea with tannery sludge addition, however, in higher rates, the residue decreased the Bradyrhizobium sp nodulation.
\end{abstract}

Index terms: Bradyrhizobium; biological indicator; Vigna unguiculata.

(Recebido para publicação em 3 de Junho de 2005 e aprovado em 15 de maio de 2006)

\section{INTRODUÇÃO}

$\mathrm{O}$ aumento da atividade humana e industrial traz como consequiência a geração cada vez maior de resíduos orgânicos, principalmente lodo de esgoto, lixo urbano e resíduos industriais. A utilização destes resíduos como fonte de nutrientes para as plantas e condicionadores dos solos constitui-se em uma alternativa viável na preservação da qualidade ambiental (ARAÚJO, 2004). Os curtumes produzem resíduos com elevadas cargas orgânica e inorgânica, além do cromo trivalente $\left(\mathrm{Cr}^{3+}\right)$, utilizado no processo de curtimento (CASTILHOS et al., 2002). Costa et al. (2001) destacam que o uso destes resíduos em áreas agrícolas, condicionando as propriedades do solo e servindo de fonte de nutrientes essenciais para as plantas, deve ter critérios definidos de aplicaçao, evitando prejuízos ao meio ambiente.

O lodo de curtume apresenta efeito relativamente pequeno sobre os microrganismos do solo (KONRAD \& CASTILHOS, 2001). Alguns trabalhos foram realizados avaliando o efeito do lodo de curtume sobre a atividade e a população microbiana do solo (ANDRÉ \& MATTIAZZO, 1997; KONRAD \& CASTILHOS, 2001). Entretanto, até o momento, nenhum estudo foi realizado verificando o efeito do lodo de curtume sobre a nodulação e a fixação biológica do nitrogênio (FBN) em leguminosas, embora outros trabalhos já tenham sido realizados utilizando a nodulação e a FBN para verificar o efeito de resíduos orgânicos, tais como lodo de esgoto (FERREIRA \& CASTRO, 1995; VIEIRA, 2001) e lodo têxtil (ARAÚJO, 2004) sobre o solo. A nodulação e a FBN vêm sendo incluídas entre os mais importantes indicadores biológicos de poluição do solo (VISER \& PARKINSON, 1992).

Neste contexto, o presente trabalho teve como objetivo avaliar o efeito da adição de lodo de curtume sobre a fertilidade do solo, nodulação e rendimento de matéria seca em caupi (Vigna unguiculata L. Walp).

\section{MATERIAL E MÉTODOS}

$\mathrm{O}$ experimento foi conduzido em casa-de-vegetação do Centro de Ciências Agrárias da Universidade Federal

\footnotetext{
${ }^{1}$ Acadêmico em Engenharia Agronômica - Centro de Ciências Agrárias - Universidade Federal do Piauí/UFPI - Campus da Socopo - Teresina, PI. ${ }^{2}$ Engenheira Agrônoma , MsC. - Centro de Ciências Agrárias - Universidade Federal do Piauí/UFPI - Teresina, PI - eulaliac@bol.com.br ${ }^{3}$ Engenheiro Agrônomo, Dr. - Universidade Federal do Piauí- Campus de Bom Jesus - Bom Jesus,PI - 64970-220 - asfarauj@yahoo.com.br ${ }^{4}$ Engenheiro Agrônomo, MsC. - Universidade Estadual do Piauí/UESPI - Campus de Parnaíba - Parnaíba, PI - santosvb@bol.com.br
} 
do Piauí (CCA/UFPI), utilizando-se um Neossolo Flúvico (RU) coletado na profundidade de $0-15 \mathrm{~cm}$, em 20 pontos inteiramente ao acaso, de uma área do CCA/UFPI. O lodo utilizado foi coletado em um curtume localizado no município de Teresina, PI, sendo em seguida colocado para secar e peneirado em malha de $2 \mathrm{~mm}$. As características químicas do solo e do lodo de curtume, determinadas conforme metodologia descrita por Tedesco et al. (1995), são apresentadas na Tabela 1. Os tratamentos estudados foram: Testemunha (T1); NPK (T2); Inoculação com Bradyrhizobium sp. + PK (T3); Lodo de curtume (11.625 $\left.\mathrm{kg} \mathrm{ha}^{-1}\right)+$ Inoculação + PK (T4); Lodo de curtume (23.250 $\left.\mathrm{kg} \mathrm{ha}^{-1}\right)+$ Inoculação + PK (T5); Lodo de curtume (46.500 $\left.\mathrm{kg} \mathrm{ha}^{-1}\right)+$ Inoculação + PK (T6). A adubação foi realizada no plantio com a aplicação de $100 \mathrm{~kg}$ de N, na forma de uréia, $80 \mathrm{~kg} \mathrm{de} \mathrm{P}_{2} \mathrm{O}_{5}$, na forma de superfosfato simples e 60 $\mathrm{kg}$ de $\mathrm{K}_{2} \mathrm{O}$, na forma de cloreto de potássio, por hectare.

As quantidades de lodo de curtume foram aplicadas de modo a incorporar ao solo 250, 500 e $1000 \mathrm{mg} \mathrm{Cr} \mathrm{kg}^{-1}$, equivalentes à aplicação de $11.625,23.250$ e $46.500 \mathrm{~kg} \mathrm{ha}^{-1}$. Foram utilizados sacos contendo $5 \mathrm{~kg}$ de solo, onde foram plantadas seis sementes da cultivar BRS-Paraguaçu de caupi inoculadas com a estirpe de Bradyrhizobium sp. BR2001 na taxa de $1 \mathrm{~kg}$ de inoculante por $40 \mathrm{~kg}$ de sementes. Aos sete dias após a emergência das plantas (DAE) foi realizado o desbaste, deixando-se 2 plantas por saco. Os dados foram coletados aos 35 e $49 \mathrm{DAE}$, quando as plantas foram cortadas próximo à base do caule e secadas em estufa a $65^{\circ} \mathrm{C}$, para determinação da massa seca da parte aérea por gravimetria. Os nódulos foram destacados das raízes, contados, secos em estufa a $65^{\circ} \mathrm{C}$ e pesados. Ao final do experimento, o solo foi removido dos vasos e analisado em laboratório (TEDESCO et al., 1995). O delineamento experimental utilizado foi inteiramente casualizado com três repetições. Os dados obtidos foram submetidos à análise de variância e as médias comparadas pelo teste de Duncan, a $5 \%$ de probabilidade.

\section{RESULTADOS E DISCUSSÃO}

\section{Alterações na fertilidade do solo}

Nos tratamentos com fertilização mineral, mas sem adição do lodo de curtume (T2 e T3), houve uma diminuição significativa do $\mathrm{pH}$ do solo após o cultivo do caupi. Por outro lado, nos tratamentos com lodo (T4, T5 e T6) houve uma elevação do pH (Tabela 2). Estes resultados indicam que os fertilizantes minerais contribuíram para a acidificação do solo, enquanto que o lodo de curtume, por ser um material alcalino (Tabela 1), influenciou a elevação do $\mathrm{pH}$ do solo. Resultados semelhantes foram relatados por Costa et al. (2001) e Ferreira et al. (2003) que verificaram a eficiência do lodo de curtume na elevação do pH do solo.

A adição do lodo de curtume nas taxas de 23.250 (T5) e 46.500 (T6) $\mathrm{kg} \mathrm{ha}^{-1}$ proporcionou a manutenção da acidez potencial do solo $(\mathrm{H}+\mathrm{Al})$ em valores próximos ao da instalação do experimento (Tabelas 1 e 2). Houve aumentos significativos, de 1,80 a 3,35 vezes, nos teores de matéria orgânica do solo (MOS) com a adição das maiores doses do lodo (T5 e T6), comparado ao conteúdo de MOS antes da instalação do experimento (Tabelas 1 e 2). Esse aporte de matéria orgânica proporcionado pela adição do lodo de curtume é de extrema importância para a região semi-árida nordestina que, naturalmente, apresenta baixos teores de MOS. Entretanto, o lodo de curtume deve ser utilizado com critérios definidos, de modo a não causar poluição ambiental e prejuízos à saúde humana e animal (COSTA et al., 2001).

TABELA 1 - Caracterização química do solo, antes do experimento, e do lodo de curtume utilizado.

\begin{tabular}{|c|c|c|}
\hline Determinação & Solo & Lodo de curtume \\
\hline $\mathrm{pH}_{\text {água }}(1: 2,5)$ & 6,53 & 7,7 \\
\hline Matéria orgânica & $13,34 \mathrm{~g} \mathrm{~kg}^{-1}$ & $407,4 \mathrm{~g} \mathrm{~kg}^{-1}$ \\
\hline $\mathrm{N}$ & & $22,9 \mathrm{~g} \mathrm{~kg}^{-1}$ \\
\hline $\mathrm{Ca}^{2+}$ & $2,74 \mathrm{cmol}_{\mathrm{c}} \mathrm{dm}^{-3}$ & $46,4 \mathrm{~g} \mathrm{~kg}^{-1}$ \\
\hline $\mathrm{Mg}^{2+}$ & $0,99 \mathrm{cmol}_{\mathrm{c}} \mathrm{dm}^{-3}$ & $27,2 \mathrm{~g} \mathrm{~kg}^{-1}$ \\
\hline $\mathrm{Na}^{+}$ & $0,06 \mathrm{cmol}_{\mathrm{c}} \mathrm{dm}^{-3}$ & $4,2 \mathrm{~g} \mathrm{~kg}^{-1}$ \\
\hline $\mathrm{K}^{+}$ & $0,31 \mathrm{cmol}_{\mathrm{c}} \mathrm{dm}^{-3}$ & $6,4 \mathrm{~g} \mathrm{~kg}^{-1}$ \\
\hline $\mathrm{P}$ & $49,03 \mathrm{mg} \mathrm{dm}^{-3}$ & $1,3 \mathrm{~g} \mathrm{~kg}^{-1}$ \\
\hline $\mathrm{Al}^{3+}+\mathrm{H}^{+}$ & $0,61 \mathrm{cmol}_{\mathrm{c}} \mathrm{dm}^{-3}$ & - \\
\hline $\mathrm{Cr}$ & - & $43,0 \mathrm{~g} \mathrm{~kg}^{-1}$ \\
\hline
\end{tabular}

Ciênc. agrotec., Lavras, v. 30, n. 6, p. 1071-1076, nov./dez., 2006 
TABELA 2 - Caracterização química do solo ao final do experimento de nodulação do feijão-caupi (49 dias após a emergência).

\begin{tabular}{|c|c|c|c|c|c|c|c|c|c|}
\hline Tratamentos* & pH & MO & $\mathrm{Ca}^{2+}$ & $\mathrm{Mg}^{2+}$ & $\mathrm{Na}^{+}$ & $\mathrm{Al}^{3+}+\mathrm{H}^{+}$ & $\mathbf{K}^{+}$ & $\mathbf{P}$ & $\mathbf{C E} * *$ \\
\hline & & $\mathrm{g} \mathrm{kg}^{-1}$ & \multicolumn{4}{|c|}{ - } & \multicolumn{2}{|c|}{$--\mathrm{mg} \mathrm{kg}^{-1}--$} & $\mathrm{dS} \mathrm{m}^{-1}$ \\
\hline $\mathrm{T} 1$ & $7,0 a b$ & $8,0 \mathrm{~b}$ & $2,1 \mathrm{~b}$ & 0,7 & $0,19 \mathrm{~b}$ & $2,6 \mathrm{a}$ & $66 \mathrm{ab}$ & $47 \mathrm{~b}$ & $0,2 \mathrm{~b}$ \\
\hline $\mathrm{T} 2$ & $6,7 \mathrm{~b}$ & $7,9 \mathrm{~b}$ & $2,9 \mathrm{~b}$ & 0,7 & $0,18 \mathrm{~b}$ & $2,3 \mathrm{a}$ & $54 \mathrm{~b}$ & $70 \mathrm{ab}$ & $0,2 \mathrm{~b}$ \\
\hline T3 & $6,5 \mathrm{~b}$ & $7,3 \mathrm{~b}$ & $2,3 \mathrm{~b}$ & 0,8 & $0,23 \mathrm{~b}$ & $1,7 \mathrm{ab}$ & $74 \mathrm{a}$ & 93 a & $0,4 \mathrm{~b}$ \\
\hline $\mathrm{T} 4$ & $7,4 a b$ & $10,9 \mathrm{~b}$ & $3,4 \mathrm{~b}$ & 0,4 & $0,23 \mathrm{~b}$ & $1,5 \mathrm{ab}$ & $74 \mathrm{a}$ & $78 \mathrm{a}$ & $0,4 \mathrm{~b}$ \\
\hline T5 & $7,5 \mathrm{a}$ & $37,4 \mathrm{a}$ & $6,7 \mathrm{a}$ & 1,1 & $0,35 \mathrm{ab}$ & $0,7 \mathrm{~b}$ & $74 \mathrm{a}$ & $88 \mathrm{a}$ & $0,7 \mathrm{ab}$ \\
\hline T6 & $7,7 \mathrm{a}$ & $58,0 \mathrm{a}$ & $7,1 \mathrm{a}$ & 1,4 & $0,58 \mathrm{a}$ & $0,7 \mathrm{~b}$ & $89 \mathrm{a}$ & $54 \mathrm{~b}$ & $1,0 \mathrm{a}$ \\
\hline
\end{tabular}

*T1 = Testemunha; T2 = adubação com N, P e K; T3 = Inoculação com Bradyrhizobium sp. + PK;T4 = Lodo de curtume $\left(11.625 \mathrm{~kg} \mathrm{ha}^{-1}\right)+$ Inoculação + PK; T5 = Lodo de curtume $\left(23.250 \mathrm{~kg} \mathrm{ha}^{-1}\right)+$ Inoculação + PK; T6 = Lodo de curtume $\left(46.500 \mathrm{~kg} \mathrm{ha}^{-1}\right)+$ Inoculação + PK.

**Condutividade elétrica.

***Médias de 3 repetições quando seguidas pela mesma letra não diferem entre si pelo teste de Duncan ao nível de 5\%.

Os teores de $\mathrm{Ca}^{2+}$ disponível no solo, após o cultivo do caupi, aumentaram nos tratamentos com a adição do lodo, apresentando um aumento de 1,6 vezes, na dose mais elevada (T6). Este aumento de $\mathrm{Ca}^{2+}$ no solo pode ser devido ao elevado conteúdo do elemento no resíduo (Tabela 1), que normalmente é encontrado nas formas de sulfeto e hidróxido (FERREIRA et al., 2003). Resultados semelhantes foram observados por Castilhos et al. (2002), Ferreira et al. (2003) e Selbach et al. (1991), em solos de clima temperado. Em relação aos teores de $\mathrm{Mg}^{2+}$, não foram observadas diferenças significativas entre os tratamentos.

Houve uma diminuição nos teores de P disponível no solo com a adição da dose mais elevada do lodo (T6), sendo semelhante, inclusive, à testemunha (T1), como pode ser observado na Tabela 2. Provavelmente, a adição da dose mais elevada contribuiu para precipitação do $\mathrm{P}$ pelo aumento do pH do solo, pois segundo Gianello et al. (1995) e Malavolta (1967) em pH elevado ocorre a precipitação do $\mathrm{P}$ em formas insolúveis de fosfato de cálcio e hidroxiapatita. Quanto aos teores de K no solo, não foram observadas diferenças significativas entre os tratamentos com adição do lodo de curtume e a testemunha.

Os tratamentos com as maiores taxas de lodo de curtume (T5 e T6) apresentaram maiores valores da condutividade elétrica do extrato de saturação $\left(\mathrm{CE}_{\mathrm{es}}\right)$, possivelmente, devido à elevada concentração de $\mathrm{Na}^{+}$no resíduo o que proporcionou aumento nos teores do elemento no solo. Resultados semelhantes foram encontrados por Aquino Neto \& Camargo (2000) e Costa et al. (2001). Desta forma, aplicações sucessivas do lodo

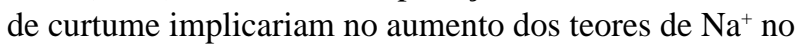

solo e, conseqüentemente, em sua salinização. Este problema pode ser minimizado com o uso de condicionadores minerais, tais como gesso agrícola $\left(\mathrm{CaSO}_{4} \cdot 2 \mathrm{H}_{2} \mathrm{O}\right)$, de modo a inativar o $\mathrm{Na}^{+}$por reação com o $\mathrm{SO}_{4}^{-}$, com posterior eliminação por lixiviação (MALAVOLTA, 1967).

Embora não tenha sido analisado devido a aspectos técnicos, a presença de $\mathrm{Cr}^{3+}$ solúvel provavelmente não seria detectada no solo. Nas condições experimentais do estudo, as amostras do solo apresentaram o valor de $\mathrm{pH}$ acima de 6,5 ao final do experimento (Tabela 2), o que, neste caso, torna o elemento insolubilizado no solo. De acordo com Bartlett \& James (1988), o $\mathrm{Cr}^{3+}$ presente no lodo de curtume é solúvel somente em $\mathrm{pH}$ do solo abaixo de 5,0, pois acima deste valor de $\mathrm{pH}$ o elemento é precipitado em formas insolúveis de $\mathrm{Cr}(\mathrm{OH})_{3} \cdot \mathrm{nH}_{2} \mathrm{O}$ no solo (AQUINO NETO \& CAMARGO, 2000).

\section{Nodulação e rendimento de matéria seca}

A adição do lodo de curtume, nas taxas de 11.625 (T4) e 23.250 (T5) kg ha-1, não apresentou efeitos negativos sobre o número de nódulos nas duas avaliações, quando comparados ao tratamento inoculado com Bradyrhizobium sp. (T3) (Tabela 3). Para a massa dos nódulos secos, houve diminuição significativa, aos 35 dias, com a adição das taxas de 23.250 (T5) e 46.500 (T6) $\mathrm{kg} \mathrm{ha}^{-1}$. Não houve diminuição no tamanho médio dos nódulos com a adição do lodo de curtume na taxa de $11.625 \mathrm{~kg} \mathrm{ha}^{-1}$ (T4), nas duas avaliações. Por outro lado, a adição da maior taxa do lodo provocou decréscimos significativos para todas as variáveis de nodulação (Tabela 3). 
O número, a massa e o tamanho dos nódulos são indicadores usuais de nodulação (FERREIRA \& CASTRO, 1995) e a presença de nódulos menores e em pequena quantidade pode ser um indicativo de condições ambientais adversas (VARGAS et al., 2004). Os resultados observados indicam que a adição do lodo de curtume ao solo, em doses elevadas, apresentou efeitos negativos sobre a nodulação pela bactéria inoculada, provavelmente devido ao aumento do teor de $\mathrm{Na}^{+}$ $\left(0,58 \mathrm{cmol}_{\mathrm{c}} \mathrm{kg}^{-1}\right)$ e da $\mathrm{CE}_{\mathrm{es}}$ do solo $\left(1,0 \mathrm{dS} \mathrm{m}^{-1}\right)$ após a adição do resíduo (Tabela 2). Embora, este valor de $\mathrm{CE}_{\text {es }}$ esteja abaixo do considerado prejudicial à simbiose Rhizobium-leguminosas (BALASUMBRAMANIAM \& SINHA, 1976), o processo de infecção bacteriana é altamente sensível à salinidade devido, principalmente, ao cessamento do crescimento do pêlo radicular (FERREIRA\& CASTRO, 1995). A nodulação da soja é totalmente suprimida em $\mathrm{CE}_{\mathrm{es}}$ superiores a $8 \mathrm{dS} \mathrm{m}^{-1}$ (SINGLETON \& BOHLOOL, 1984). Entretanto, segundo esses autores, a nodulação decresce a partir de $\mathrm{CE}_{\mathrm{es}}$ superiores a $1,0 \mathrm{dS} \mathrm{m}^{-1}$.

Na primeira avaliação (35 DAE), os rendimentos de matéria seca do caupi dos tratamentos com aplicação do lodo de curtume (T4, T5 e T6), adubação com NPK (T2) e inoculação com Bradyrhizobium sp (T3) foram estatisticamente iguais (Tabela 4), com menor rendimento obtido pela testemunha (T1).

TABELA 3 - Número, massa seca e tamanho médio dos nódulos do caupi cultivado em solo com diferentes tratamentos de lodo de curtume e inoculação.

\begin{tabular}{|c|c|c|c|c|c|c|}
\hline \multirow[b]{2}{*}{ Tratamento* } & \multicolumn{2}{|c|}{$\begin{array}{c}\text { Número de nódulos } \\
\left(n^{0} \text { vaso }^{-1}\right)\end{array}$} & \multicolumn{2}{|c|}{$\begin{array}{c}\text { Massa dos nódulos secos } \\
\left(\mathrm{mg} \mathrm{vaso}^{-1}\right)\end{array}$} & \multicolumn{2}{|c|}{$\begin{array}{l}\text { Tamanho médio dos } \\
\text { nódulos (mg nódulo }{ }^{-1} \text { ) }\end{array}$} \\
\hline & 35 & 49 & 35 & 49 & 35 & 49 \\
\hline $\mathrm{T} 1$ & $26,6 \mathrm{bc}$ & $65,0 \mathrm{abc}$ & $83,0 \mathrm{bc}$ & $162,3 \mathrm{ab}$ & $3,0 \mathrm{a}$ & $4,9 \mathrm{abc}$ \\
\hline $\mathrm{T} 2$ & $33,3 \mathrm{bc}$ & $67,0 a b c$ & $65,6 \mathrm{bc}$ & $175,6 \mathrm{a}$ & $1,7 \mathrm{bc}$ & $5,0 \mathrm{ab}$ \\
\hline T3 & $53,0 \mathrm{a}$ & $78,3 \mathrm{a}$ & $153,0 \mathrm{a}$ & 203,6 a & $2,9 \mathrm{ab}$ & $5,2 \mathrm{a}$ \\
\hline $\mathrm{T} 4$ & $43,3 \mathrm{ab}$ & $73,3 \mathrm{ab}$ & $112,0 \mathrm{ab}$ & $166,3 \mathrm{a}$ & $2,6 \mathrm{abc}$ & $4,4 a b c$ \\
\hline $\mathrm{T} 5$ & $39,3 \mathrm{abc}$ & $73,6 \mathrm{ab}$ & $78,6 \mathrm{bc}$ & $142,5 \mathrm{ab}$ & $2,1 \mathrm{abc}$ & $3,8 \mathrm{bc}$ \\
\hline T6 & $22,6 \mathrm{c}$ & $47,0 \mathrm{c}$ & $26,7 \mathrm{c}$ & $104,3 \mathrm{~b}$ & $1,3 \mathrm{c}$ & $3,7 \mathrm{c}$ \\
\hline
\end{tabular}

*T1 = Testemunha; T2 = adubação com N, P e K; T3 = Inoculação com Bradyrhizobium sp. + PK; T4 = Lodo de curtume $\left(11.625 \mathrm{~kg} \mathrm{ha}^{-1}\right)+$ Inoculação + PK; T5 = Lodo de curtume $\left(23.250 \mathrm{~kg} \mathrm{ha}^{-1}\right)+$ Inoculação + PK; T6 = Lodo de curtume $\left(46.500 \mathrm{~kg} \mathrm{ha}^{-1}\right)+$ Inoculação + PK.

***Dias após a emergência.

Médias de 3 repetições quando seguidas da mesma letra não diferem entre si pelo teste de Duncan ao nível de 5\%.

TABELA 4 - Rendimento de matéria seca da parte aérea do caupi cultivado em solo com diferentes tratamentos de lodo de curtume e inoculação.

\begin{tabular}{ccc}
\hline & \multicolumn{2}{c}{${\text { Matéria seca da parte aérea }\left(\text { g vaso }^{-1}\right)}$} \\
\hline Tratamento* & DAE** $^{* *}$ & $\mathbf{4 9}$ \\
\hline T1 & & \\
T2 & $2,93 \mathrm{~b}$ & $7,96 \mathrm{a}$ \\
T3 & $5,02 \mathrm{a}$ & $8,32 \mathrm{a}$ \\
T4 & $3,81 \mathrm{ab}$ & $7,79 \mathrm{a}$ \\
T5 & $3,77 \mathrm{ab}$ & $7,69 \mathrm{a}$ \\
T6 & $3,81 \mathrm{ab}$ & $8,11 \mathrm{a}$ \\
& $3,59 \mathrm{ab}$ & $10,51 \mathrm{a}$ \\
\hline
\end{tabular}

*T1 = Testemunha; T2 = adubação com N, P e K; T3 = Inoculação com Bradyrhizobium sp. + PK;T4 = Lodo de curtume $\left(11.625 \mathrm{~kg} \mathrm{ha}^{-1}\right)+$ Inoculação + PK; T5 = Lodo de curtume $\left(23.250 \mathrm{~kg} \mathrm{ha}^{-1}\right)+$ Inoculação + PK; T6 = Lodo de curtume $\left(46.500 \mathrm{~kg} \mathrm{ha}^{-1}\right)+$ Inoculação + PK.

***Dias após a emergência.

Médias de 3 repetições quando seguidas da mesma letra não diferem entre si pelo teste de Duncan ao nível de 5\%.

Ciênc. agrotec., Lavras, v. 30, n. 6, p. 1071-1076, nov./dez., 2006 
Na segunda avaliação (49 DAE) não houve diferenças entre os tratamentos, embora o tratamento com a adição do lodo de curtume na taxa de $46.500 \mathrm{~kg} \mathrm{ha}^{-1}$ tenha apresentado um incremento no rendimento de matéria seca. Estes resultados foram semelhantes aos encontrados por Ferreira et al. (2003) em plantas de milho e soja, e indicam que o lodo de curtume proporcionou a liberação de nutrientes para o caupi, uma vez que os teores de matéria orgânica $\left(58 \mathrm{~g} \mathrm{~kg}^{-1}\right)$ e $\mathrm{Ca}^{+2}\left(7,1 \mathrm{cmol}_{\mathrm{c}} \mathrm{kg}^{-1}\right)$ no solo aumentaram após a adição da maior taxa do resíduo.

\section{CONCLUSÕES}

A adição do lodo de curtume elevou o pH e os teores de matéria orgânica, cálcio e sódio do solo. Em altas doses, o resíduo pode aumentar o nível de salinidade do solo.

O lodo de curtume, em altas doses, diminui a nodulação do caupi pela estirpe de Bradyrhizobium $s p$ inoculada.

\section{REFERÊNCIAS BIBLIOGRÁFICAS}

ANDRÉ, E. M.; MATTIAZZO, M. E. Biodegradabilidade de um resíduo de curtume aplicado a latossolos. In: CONGRESSO BRASILEIRO DE CIENCIA DO SOLO, 26., 1997, Rio de Janeiro. Resumos Expandidos... Rio de Janeiro: SBCS, 1997. CD-ROM.

AQUINO NETO, V.; CAMARGO, O. A. Crescimento e acúmulo de cromio em alface cultivada em dois latossolos tratados com $\mathrm{CrCl}_{3}$ e resíduos de curtume. Revista Brasileira de Ciência do Solo, Campinas, v. 24, p. 225-235, 2000.

ARAÚJO, A. S. F. A compostagem do lodo têxtil e seu efeito sobre indicadores biológicos. 2004. 89 f. Tese (Doutorado em Ecologia de Agroecossistemas) - Escola Superior de Agricultura Luiz de Queiroz, Piracicaba, 2004.

BALASUMBRAMANIAM, V.; SINHA, S. K. Effects of salt stress on growth, nodulation and nitrogen fixation in cowpea and mungbean. Plant Physiology, Berlin, v. 36, p. 179-200, 1976.

BARTLETT, R. J.; JAMES, B. R. Mobility and bioavailability of chromium in soils. In: NRIAGU, J. O.; NIEBOER, E. Chromium in the natural and human environments. New York: J. Wiley \& Sons, 1988. p. 267304.

CASTILHOS, D. D.; TEDESCO, M. J.; VIDOR, C. Rendimentos de culturas e alterações químicas do solo tratado com resíduos de curtume e cromio hexavalente. Revista Brasileira de Ciência do Solo, Viçosa, v. 26, p. 1083-1092, 2002.

COSTA, C. N.; CASTILHOS, D. D.; CASTILHOS, R. M. V.; KONRAD, E. E.; PASSIANOTO, C. C.; RODRIGUES, C. G. Efeito de adição de lodo de curtume sobre as alterações químicas do solo, rendimento de matéria seca e absorção de nutrientes em soja. Revista Brasileira de Agrociência, Pelotas, v. 7, p. 189-191, 2001.

FERREIRA, A. S.; CAMARGO, F. A. O.; TEDESCO, M. J.; BISSANI, C. A. Alterações de atributos químicos e biológicos de solo e rendimento de milho e soja pela utilização de resíduos de curtume e carbonífero. Revista Brasileira de Ciência do Solo, Viçosa, v. 27, p. 755-763, 2003.

FERREIRA, E. M.; CASTRO, I. V. Nodulation and growth of subterranean clover (Trifolium subterraneum L.) in soils previously treated with sewage sludge. Soil Biology and Biochemistry, Oxford, v. 27, p. 1177-1183, 1995.

GIANELLO, C.; BISSANI, C. A.; TEDESCO, M. J. Princípios de fertilidade do solo. Porto Alegre: UFRGS, 1995. $276 \mathrm{p}$.

KONRAD, E. E.; CASTILHOS, D. D. Atividade microbiana em um Planossolo após a adição de resíduos de curtume. Revista Brasileira de Agrociência, Pelotas, v. 7, p. 131-135, 2001.

MALAVOLTA, E. Manual de química agrícola: adubos e adubações. 2. ed. São Paulo: Ceres, 1967. 606 p.

SELBACH, P. A.; TEDESCO, M. J.; GIANELLO, C. Descarte e biodegradação de lodo de curtume no solo. Revista Couro, São Paulo, v. 4, p. 51-62, 1991.

SINGLETON, P. W.; BOHLOOL, B. B. Effect of salinity on nodule formation by soybean. Plant Physiology, Berlin, v. 74, p. 72-76, 1984. 
TEDESCO, M. J.; GIANELLO, C.; BISSANI, C. A. Análises de solos, plantas e outros materiais. Porto Alegre: UFRGS, 1995. $174 \mathrm{p}$.

VARGAS, M. A. T.; MENDES, I. C.; CARVALHO, A. M.; LOBOBURLE, M.; HUNGRIA, M. Inoculação de leguminosas e manejo de adubos verdes. In: SOUSA, D. M. G.; LOBATO, E. Cerrado: correção do solo e adubação. Brasília, DF: Embrapa, 2004. p. 97-128.
VIEIRA, R. F. Sewage sludge effects on soybean growth and nitrogen fixation. Biology and Fertility of Soils, Berlin, v. 34, p. 196-200, 2001.

VISER, S.; PARKINSON, D. Soil biological criteria as indicator of soil quality: soil microorganisms. American Journal of Alternative Agriculture, New York, v. 7, p. 3337, 1992.

Ciênc. agrotec., Lavras, v. 30, n. 6, p. 1071-1076, nov./dez., 2006 